\title{
Q-reflexive Locally Convex Spaces
}

\author{
By \\ Christopher Boyd, Seán Dineen and Milena Venkova*
}

\begin{abstract}
For a locally convex space $E$ we use the Aron-Berner extension to define canonical mappings from $\widehat{\bigotimes} E_{s, n, \pi}^{\prime \prime}$ into different duals of $\mathcal{P}\left({ }^{n} E\right)$. We investigate necessary and sufficient conditions for the continuity of these mappings, paying particular attention to three special cases - Fréchet spaces, DF spaces and reflexive A-nuclear spaces. We define Q-reflexive spaces as spaces where a certain canonical mapping can be extended to an isomorphism between $\widehat{\bigotimes_{s, n, \pi}} E_{e}^{\prime \prime}$ and $\overline{\left(\mathcal{P}\left({ }^{n} E\right), \tau_{b}\right)_{i}^{\prime}}$. We find examples of such spaces.
\end{abstract}

\section{$\S 1$. Introduction}

In [3] R. Aron and S. Dineen considered the problem of obtaining a polynomial functional representation of the bidual of the space of continuous $n$ homogeneous polynomials on a Banach space $E$. More precisely, they asked when the space $\mathcal{P}\left({ }^{n} E\right)^{\prime \prime}$ is isomorphic to $\mathcal{P}\left({ }^{n} E^{\prime \prime}\right)$ in a canonical way. Spaces with this property are called $Q$-reflexive. A reflexive Banach space $E$ with the approximation property is Q-reflexive if and only if $\mathcal{P}\left({ }^{n} E\right)$ is reflexive.

In this article we consider the analogous problem when $E$ is a locally convex space. When $E$ is a Banach space, $\mathcal{P}\left({ }^{n} E\right)$ endowed with the topology of uniform convergence over the unit ball of $E$ is a Banach space. The situation becomes complicated in the more general setting due to the increased choice of topologies on $\mathcal{P}\left({ }^{n} E\right)$ and the dual of $E$. To arrive at a suitable definition of Q-reflexive locally convex space we examine three classes of spaces which

Communicated by T. Kawai. Received October 25, 2002. Revised February 17, 2003.

2000 Mathematics Subject Classification(s): 46G25, 46A20, 46A25, 46A04

* Department of Mathematics, University College Dublin, Belfield, Dublin 4, Ireland. 
have shown themselves to be interesting from polynomial and holomorphic viewpoints - Fréchet spaces, DF spaces and fully nuclear spaces. We refer to [11] and [15] for background information on polynomials over locally convex spaces and the theory of locally convex spaces respectively.

\section{$\S 2 . \quad$ Biduals of Spaces of Homogeneous Polynomials with the Compact Open Topology}

In this section we discuss spaces of polynomials endowed with the compact open topology $\tau_{0}$. Biduality, when the domain space is either DF or Fréchet, is relatively straightforward in this case. We first, however, introduce some notation that will be used throughout the article. Let $E$ be a locally convex space over the complex numbers $\mathbb{C}$. We will denote by $\bar{E}$ the completion of $E$, and by $E^{\prime}$ the space of all continuous linear functionals on $E$. If $E^{\prime}$ is endowed with the strong topology (i.e. the topology of uniform convergence over the bounded subsets of $E$ ) we denote it by $E_{\beta}^{\prime}$. We say that $E$ is infrabarrelled (or quasibarrelled) if the canonical inclusion of $E$ into $E_{\beta \beta}^{\prime \prime}:=\left(E_{\beta}^{\prime}\right)_{\beta}^{\prime}$ is continuous. Let $\mathcal{V}$ be a fundamental 0-neighbourhood basis of $E$, the collection $\left(V^{\circ 0}\right)_{V \in \mathcal{V}}$ is a fundamental 0-neighbourhood basis for the natural topology on $E^{\prime \prime}$. The bidual of $E$ endowed with the natural topology is denoted by $E_{e}^{\prime \prime}$. It is well known that $E$ is infrabarrelled if and only if $E_{e}^{\prime \prime}=E_{\beta \beta}^{\prime \prime}$, or, equivalently, if and only if the bounded subsets of $E_{\beta}^{\prime}$ are equicontinuous. A locally convex space $E$ is barrelled if and only if the $\sigma\left(E^{\prime}, E\right)$-bounded subsets in $E^{\prime}$ are equicontinuous (thus every barrelled space is infrabarrelled). A locally convex space is distinguished if its strong dual is barrelled.

For $E$ a locally convex space we let $\mathcal{P}_{a}\left({ }^{n} E\right)$ denote the vector space of all $n$ homogeneous polynomials on $E$, and $\mathcal{P}\left({ }^{n} E\right)$ denote the space of all continuous $n$-homogeneous polynomials on $E$. The topology on $\mathcal{P}\left({ }^{n} E\right)$ of uniform convergence over the compact (respectively bounded) subsets of $E$ is denoted by $\tau_{0}$ (respectively $\left.\tau_{b}\right)$. A third topology on $\mathcal{P}\left({ }^{n} E\right)$ can be defined in the following way. A semi-norm $p$ on $\mathcal{P}\left({ }^{n} E\right)$ is $\tau_{\omega}$-continuous if for every zero neighbourhood $V$ in $E$ there exists a positive constant $C(V)$ such that

$$
p(P) \leq C(V)\|P\|_{V}
$$

for all $P \in \mathcal{P}\left({ }^{n} E\right)$. The topology generated by all such semi-norms is denoted by $\tau_{\omega}$. When $n=1, E_{i}^{\prime}:=\left(\mathcal{P}\left({ }^{1} E\right), \tau_{\omega}\right)$ is the inductive dual of $E$, $E_{\beta}^{\prime}:=\left(\mathcal{P}\left({ }^{1} E\right), \tau_{b}\right)$ is the strong dual of $E$ and, if $E$ is quasi-complete, $E_{c}^{\prime}:=$ $\left(\mathcal{P}\left({ }^{1} E\right), \tau_{0}\right)$. 
If $\widehat{\bigotimes_{s, n, \pi}} E$ denotes the completed symmetric $n$-fold tensor product of $E$

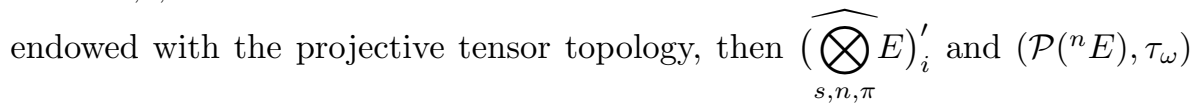
are isomorphic. The space $E$ has the $(B B)_{n}$ property if the closed convex hull of $\otimes_{n, s} B$ forms a fundamental system of bounded subsets of $\widehat{\bigotimes_{s, n, \pi}} E$ as $B$ ranges over the bounded subsets of $E$. Clearly $E$ has $(B B)_{n}$ if and only if $\left(\widehat{\bigotimes_{s, n, \pi}} E\right)_{\beta}^{\prime}$ and $\left(\mathcal{P}\left({ }^{n} E\right), \tau_{b}\right)$ are isomorphic. A locally convex space in which all closed bounded sets are compact is called semi-Montel. A semi-Montel Fréchet space is called Fréchet-Montel and a semi-Montel DF space is called a DFM space.

Proposition 2.1. $\quad$ Let $E$ be a Fréchet space and $n$ a positive integer. Then

(a) $\left(\left(\mathcal{P}\left({ }^{n} E\right), \tau_{0}\right)_{\beta}^{\prime}\right)_{\beta}^{\prime}=\left(\mathcal{P}\left({ }^{n} E\right), \tau_{w}\right)$ if and only if $\widehat{\bigotimes_{s, n, \pi}} E$ is a distinguished Fréchet space.

(b) $\left(\left(\mathcal{P}\left({ }^{n} E\right), \tau_{0}\right)_{\beta}^{\prime}\right)_{\beta}^{\prime}=\left(\mathcal{P}\left({ }^{n} E\right), \tau_{0}\right)$ if and only if $E$ is a Fréchet-Montel space with the $(B B)_{n}$ property.

Proof. By ([11], Proposition 2.20),

$$
\left(\left(\mathcal{P}\left({ }^{n} E\right), \tau_{0}\right)_{\beta}^{\prime}\right)_{\beta}^{\prime}=\left(\widehat{\bigotimes_{s, n, \pi} E}\right)_{\beta}^{\prime} .
$$

Hence (a) holds if and only if $\left(\widehat{\bigotimes_{s, n, \pi}} E\right)_{i}^{\prime}=\left(\widehat{\bigotimes_{s, n, \pi}} E\right)_{\beta}^{\prime}$. Since $E$ is Fréchet $\widehat{\bigotimes} E$ is also Fréchet. As the strong and inductive duals of a Fréchet space have the same bounded sets, a result of Grothendieck ([14], Theorem 3.16.1)

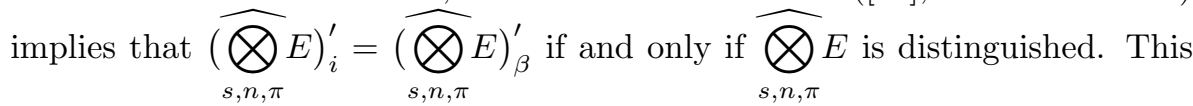
proves (a).

By $(2.1),(\mathrm{b})$ holds if and only if $\left(\widehat{\bigotimes_{s, n, \pi}} E\right)_{\beta}^{\prime}$ and $\left(\mathcal{P}\left({ }^{n} E\right), \tau_{0}\right)=\left(\widehat{\bigotimes_{s, n, \pi}} E\right)_{c}^{\prime}$ are isomorphic, i.e. if and only if $\widehat{\bigotimes_{s, n, \pi}} E$ is Fréchet-Montel. By ([11], Proposition 
1.35) and [1], $\widehat{\bigotimes}_{s, n, \pi} E$ is Fréchet-Montel space if and only if $E$ is a Fréchet-Montel space with $(B B)_{n}$. This completes the proof of (b).

Proposition 2.2. Let $E$ be a complete infrabarrelled DF space. Then (a) $\left(\left(\mathcal{P}\left({ }^{n} E\right), \tau_{0}\right)_{\beta}^{\prime}\right)_{\beta}^{\prime}=\left(\mathcal{P}\left({ }^{n} E\right), \tau_{\omega}\right)$ for every $n$.

(b) $\left(\left(\mathcal{P}\left({ }^{n} E\right), \tau_{0}\right)_{\beta}^{\prime}\right)_{\beta}^{\prime}=\left(\mathcal{P}\left({ }^{n} E\right), \tau_{0}\right)$ for every $n$ if and only if $E$ is a DFM space.

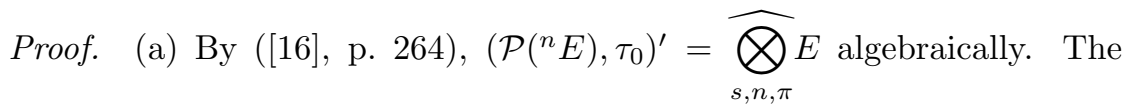
topology on $\widehat{\bigotimes_{s, n, \pi}} E$ is the topology of uniform convergence on the equicontinuous subsets of the dual $\left(\widehat{\bigotimes_{s, n, \pi}} E\right)^{\prime}=\mathcal{P}\left({ }^{n} E\right)$, while the topology on $\left(\mathcal{P}\left({ }^{n} E\right), \tau_{0}\right)_{\beta}^{\prime}$ is the topology of uniform convergence on the $\tau_{0}$-bounded, or, by ([11], Lemma $1.23)$, the $\tau_{b}$-bounded subsets of $\mathcal{P}\left({ }^{n} E\right)$. Let $E$ be infrabarrelled, by ([15]), Proposition 15.6.8) $\widehat{\bigotimes_{s, n, \pi} E}$ is infrabarrelled and hence the strongly bounded and the equicontinuous subsets of its dual $\mathcal{P}\left({ }^{n} E\right)$ coincide. Since every DF space has $(B B)_{n}$, this means that the $\tau_{b}$-bounded subsets and the equicontinuous subsets of $\mathcal{P}\left({ }^{n} E\right)$ coincide and $\left(\mathcal{P}\left({ }^{n} E\right), \tau_{0}\right)_{\beta}^{\prime}=\widehat{\bigotimes_{s, n, \pi}} E$. By $([4]$, Corollary 3.4)

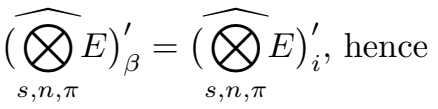

$$
\begin{aligned}
& \left(\mathcal{P}\left({ }^{n} E\right), \tau_{0}\right)_{\beta \beta}^{\prime \prime}=\left(\widehat{\bigotimes_{s, n, \pi}} E\right)_{i}^{\prime}=\left(\mathcal{P}\left({ }^{n} E\right), \tau_{\omega}\right) .
\end{aligned}
$$

This completes the proof of (a).

(b) Since $E$ is a complete infrabarrelled DF space, by (a) $\left(\left(\mathcal{P}\left({ }^{n} E\right), \tau_{0}\right)_{\beta}^{\prime}\right)_{\beta}^{\prime}=$ $\left(\mathcal{P}\left({ }^{n} E\right), \tau_{\omega}\right)$ for every $n$. Suppose $E$ is DFM, by ([11], Example 1.32) $\tau_{0}=\tau_{\omega}$ on $\mathcal{P}\left({ }^{n} E\right)$, hence $\left(\left(\mathcal{P}\left({ }^{n} E\right), \tau_{0}\right)_{\beta}^{\prime}\right)_{\beta}^{\prime}=\left(\mathcal{P}\left({ }^{n} E\right), \tau_{0}\right)$.

Conversely, suppose $\left(\left(\mathcal{P}\left({ }^{n} E\right), \tau_{0}\right)_{\beta}^{\prime}\right)_{\beta}^{\prime}=\left(\mathcal{P}\left({ }^{n} E\right), \tau_{0}\right)$. By (a), $\left(\left(\mathcal{P}\left({ }^{n} E\right)\right.\right.$, $\left.\left.\tau_{0}\right)_{\beta}^{\prime}\right)_{\beta}^{\prime}=\left(\mathcal{P}\left({ }^{n} E\right), \tau_{\omega}\right)$, so $\tau_{\omega}=\tau_{0}$ on $\mathcal{P}\left({ }^{n} E\right)$. Since $\tau_{\omega} \geq \tau_{b} \geq \tau_{0}$, the HahnBanach Theorem implies that $E$ is a DFM space. This completes the proof.

Remark 1. The space $E=\underset{\lim }{\longleftarrow}\left(c_{0}\left(\Gamma^{\prime}\right),\|\cdot\|_{\Gamma^{\prime}}\right)$, where the projective limit is over all countable $\Gamma^{\prime} \subset \Gamma$ for an uncountable $\Gamma$, is a DF space which is not infrabarrelled. Nevertheless, it can be shown that $\left(\left(\mathcal{P}\left({ }^{n} E\right), \tau_{0}\right)_{\beta}^{\prime}\right)_{\beta}^{\prime}=\left(\mathcal{P}\left({ }^{n} E\right), \tau_{\omega}\right)$ for every $n$. 


\section{§3. The Canonical Map $J_{n}$}

In this section we consider $\mathcal{P}\left({ }^{n} E\right)$ endowed with the $\tau_{\omega}$ and $\tau_{b}$ topologies. If $P \in \mathcal{P}\left({ }^{n} E\right)$ let $A B_{n}(P)$ denote the Aron-Berner extension of $P$ to $E^{\prime \prime}:=\left(E_{\beta}^{\prime}\right)^{\prime}$ (see [2]). If $x^{\prime \prime} \in E^{\prime \prime}$ then there exists a bounded subset $B$ of $E$ such that

$$
\left|A B_{n}(P)\left(x^{\prime \prime}\right)\right| \leq\|P\|_{B}
$$

for all $P \in \mathcal{P}\left({ }^{n} E\right)$. Thus the mapping

$$
J_{n}: \bigotimes_{s, n, \pi} E^{\prime \prime} \longrightarrow\left(\mathcal{P}\left({ }^{n} E\right), \tau_{b}\right)^{\prime},
$$

given by $\left[J_{n}\left(\otimes_{n} x^{\prime \prime}\right)\right](P)=\left[A B_{n}(P)\right]\left(x^{\prime \prime}\right)$ for all $P \in \mathcal{P}\left({ }^{n} E\right)$ and all $x^{\prime \prime} \in E^{\prime \prime}$, and extended by linearity, is well defined. Since the topology $\tau_{\omega}$ is finer than $\tau_{b}$, the mapping $J_{n}$ is also well defined with range space $\left(\mathcal{P}\left({ }^{n} E\right), \tau_{\omega}\right)^{\prime}$.

We are interested in turning $J_{n}$ into a continuous mapping. To proceed we need to label the different topologies that we consider. The following diagram fixes our notation:

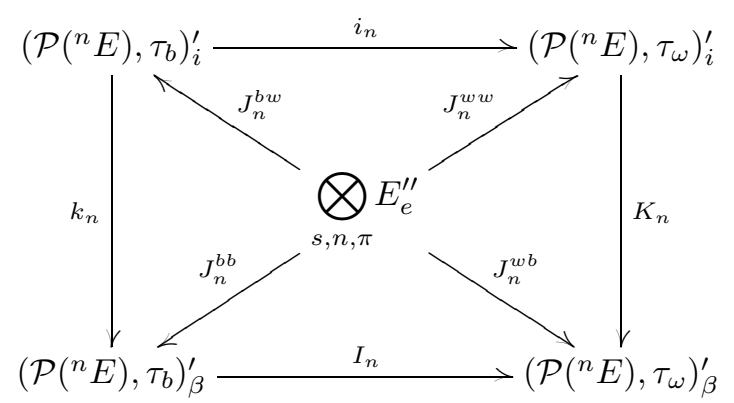

The diagonal mappings are just the mapping $J_{n}$ with superscripts used to denote the structure of the range space. The mappings along the horizontal and vertical arrows are always well defined and continuous.

Remark 2. The continuity of $J_{1}^{b b}: E_{e}^{\prime \prime} \longrightarrow\left(\mathcal{P}\left({ }^{1} E\right), \tau_{b}\right)_{\beta}^{\prime}=E_{\beta \beta}^{\prime \prime}$ implies that $E$ is infrabarrelled.

We first consider the lower diagonal mappings in Diagram (3.2).

Proposition 3.1. $\quad$ Let $E$ be a locally convex space such that the $\tau$ bounded sets of $\mathcal{P}\left({ }^{n} E\right), \tau=\tau_{b}$ or $\tau_{\omega}$, are locally bounded for some $n$. Then the 
mapping

$$
J_{n}: \bigotimes_{s, n, \pi} E_{e}^{\prime \prime} \longrightarrow\left(\mathcal{P}\left({ }^{n} E\right), \tau\right)_{\beta}^{\prime}
$$

is continuous. If $\tau=\tau_{\omega}$ then $J_{n}^{w b}$ can be extended to the completion $\widehat{\bigotimes_{s, n, \pi}} E_{e}^{\prime \prime}$.

Proof. By our hypothesis the topology on $\left(\mathcal{P}\left({ }^{n} E\right), \tau\right)_{\beta}^{\prime}$ is generated by the semi-norms

$$
\alpha_{V}(\phi)=\sup \left\{|\phi(P)|:\|P\|_{V} \leq 1\right\},
$$

where $V$ ranges over the convex balanced neighbourhoods of zero in $E$. Let $P \in$ $\mathcal{P}\left({ }^{n} E\right)$ and $\stackrel{V}{A B_{n}}(P)$ be the symmetric $n$-linear form associated with $A B_{n}(P)$. The mapping

$$
\left.j_{n}:\left(x_{1}^{\prime \prime}, \ldots, x_{n}^{\prime \prime}\right) \longrightarrow\left[P \rightarrow \stackrel{\vee}{A B} B_{n}(P)\right]\left(x_{1}^{\prime \prime}, \ldots, x_{n}^{\prime \prime}\right)\right],
$$

where $x_{i}^{\prime \prime} \in E_{e}^{\prime \prime}$ for $1 \leq i \leq n$, is symmetric, $n$-linear, and has linearization $J_{n}$. If $V$ is a convex balanced neighbourhood of zero in $E$, then by ([11], Proposition 1.53) and the Polarization Formula,

$$
\left|\left[\stackrel{\vee}{A B_{n}}(P)\right]\left(x_{1}^{\prime \prime}, \ldots, x_{n}^{\prime \prime}\right)\right| \leq \frac{n^{n}}{n !}\left\|x_{1}^{\prime \prime}\right\|_{V^{\circ \circ}} \cdots\left\|x_{1}^{\prime \prime}\right\|_{V^{\circ \circ}\|P\|_{V}}
$$

where $x_{i}^{\prime \prime} \in E_{e}^{\prime \prime}$ for $1 \leq i \leq n$ and $P \in \mathcal{P}\left({ }^{n} E\right)$. Hence

$$
\begin{aligned}
\alpha_{V}\left(j_{n}\left(x_{1}^{\prime \prime}, \ldots, x_{n}^{\prime \prime}\right)\right) & =\sup \left\{\left(\left[\stackrel{\vee}{A B_{n}}(P)\right]\left(x_{1}^{\prime \prime}, \ldots, x_{n}^{\prime \prime}\right)\right):\|P\|_{V} \leq 1\right\} \\
& \leq \frac{n^{n}}{n !}\left\|x_{1}^{\prime \prime}\right\|_{V^{\circ \circ}} \cdots\left\|x_{1}^{\prime \prime}\right\|_{V^{\circ \circ},}
\end{aligned}
$$

and $j_{n}$ is continuous. By the definition of the projective tensor product this implies that $J_{n}$ is also continuous.

When $\tau=\tau_{\omega}$ the space $\left(\mathcal{P}\left({ }^{n} E\right), \tau_{\omega}\right)_{\beta}^{\prime}$ is complete as the strong dual of a bornological space, and consequently $J_{n}$ can be extended to $\widehat{\bigotimes_{s, n, \pi}} E_{e}^{\prime \prime}$ by continuity.

Next we consider the mapping $J_{n}^{b w}$, concentrating on some special cases.

If $E$ is a Fréchet space $\left(\mathcal{P}\left({ }^{n} E\right), \tau_{\omega}\right)$ is a barrelled DF space, hence its strong and inductive duals coincide by [4]. Thus $K_{n}$ is an isomorphism and $J_{n}^{w b}=J_{n}^{w w}$ for every $n$. Moreover, the $\tau_{\omega}$-bounded and the $\tau_{b}$-bounded subsets of $\mathcal{P}\left({ }^{n} E\right)$ are locally bounded and hence, by Proposition 3.1, the mappings $J_{n}^{w b}=J_{n}^{w w}$ and $J_{n}^{b b}$ are continuous. 
Proposition 3.2. $\quad$ Let $E$ be a Fréchet space with $(B B)_{n}$ for some $n$. Then $J_{n}^{b w}$ is continuous, $k_{n}$ is an isomorphism and $J_{n}^{b w}=J_{n}^{b b}$.

Proof. Since $E$ has $(B B)_{n}$ we have

$$
\left(\mathcal{P}\left({ }^{n} E\right), \tau_{b}\right)_{i}^{\prime}=\left(\left(\widehat{\bigotimes_{s, n, \pi}} E\right)_{\beta}^{\prime}\right)_{\beta}^{\prime}=\left(\mathcal{P}\left({ }^{n} E\right), \tau_{b}\right)_{\beta}^{\prime},
$$

hence $J_{n}^{b w}=J_{n}^{b b}$ and, in particular, $J_{n}^{b w}$ is continuous. Moreover, as

$$
\left(\mathcal{P}\left({ }^{n} E\right), \tau_{b}\right)_{i}^{\prime}=\left(\mathcal{P}\left({ }^{n} E\right), \tau_{b}\right)_{\beta}^{\prime},
$$

$k_{n}$ is an isomorphism and $J_{n}^{b w}=J_{n}^{b b}$.

Next suppose $E$ is a DF space. By ([11], Example 1.32) $\tau_{b}=\tau_{\omega}$ on $\mathcal{P}\left({ }^{n} E\right)$ for every $n$, hence $i_{n}$ and $I_{n}$ are isomorphisms. Thus $J_{n}^{w b}=J_{n}^{b b}$ and $J_{n}^{b w}=J_{n}^{w w}$ for every $n$.

Proposition 3.3. Let $E$ be a DF space.

(a) The mapping

$$
J_{n}^{b b}: \bigotimes_{s, n, \pi} E_{e}^{\prime \prime} \longrightarrow\left(\mathcal{P}\left({ }^{n} E\right), \tau_{b}\right)_{\beta}^{\prime}
$$

is continuous for every $n$ if and only if $E$ is infrabarrelled. In this case $J_{n}^{b b}$ can be extended to the completion $\widehat{\bigotimes} E_{e}^{\prime \prime}=\widehat{\bigotimes, \pi}{ }_{s, n, \pi} E_{\beta \beta}^{\prime \prime}$.

(b) The mapping

$$
J_{n}^{b w}: \bigotimes_{s, n, \pi} E_{e}^{\prime \prime} \longrightarrow\left(\mathcal{P}\left({ }^{n} E\right), \tau_{b}\right)_{i}^{\prime}
$$

is continuous for every $n$ if and only if $E$ and $E_{\beta \beta}^{\prime \prime}$ are infrabarrelled. In this case $J_{n}^{b w}$ can be extended to the completion $\widehat{\bigotimes_{s, n, \pi}} E_{e}^{\prime \prime}=\widehat{\bigotimes} E_{\beta, n, \pi}^{\prime \prime}$.

Proof. (a) If $J_{n}^{b b}$ is continuous $E$ is infrabarrelled by Remark 2. Conversely, if $E$ is an infrabarrelled DF space then by ([15], Proposition 15.6.8) $\bigotimes E$ is an infrabarrelled DF space and consequently the bounded subsets $s, n, \pi$

of $\left(\bigotimes_{s, n, \pi} E\right)_{\beta}^{\prime}$ are equicontinuous. As a DF space $E$ has $(B B)_{n}$, this implies 
$\left(\bigotimes_{s, n, \pi} E\right)_{\beta}^{\prime}=\left(\mathcal{P}\left({ }^{n} E\right), \tau_{b}\right)$. By Proposition $3.1, J_{n}^{b b}$ is continuous. Since $\left(\mathcal{P}\left({ }^{n} E\right)\right.$, $\left.\tau_{b}\right)$ is Fréchet, it is bornological. Hence $\left(\mathcal{P}\left({ }^{n} E\right), \tau_{b}\right)_{\beta}^{\prime}$ is complete and the continuous mapping $J_{n}^{b b}$ can be extended to $\widehat{\bigotimes_{s, n, \pi}} E_{\beta \beta}^{\prime \prime}$.

(b) Let $E$ and $E_{\beta \beta}^{\prime \prime}$ be infrabarrelled DF spaces. As $E_{\beta \beta}^{\prime \prime}$ is the strong dual of a metrizable space, it is barrelled and bornological ([15], Corollary 13.4.4). By ([15], 15.6.8) $\bigotimes_{s, n, \pi} E_{\beta \beta}^{\prime \prime}$ is a bornological DF space. By (a) $J_{n}^{b b}$ is continuous and hence maps the bounded sets of $\bigotimes_{s, n, \pi} E_{\beta \beta}^{\prime \prime}$ onto bounded sets

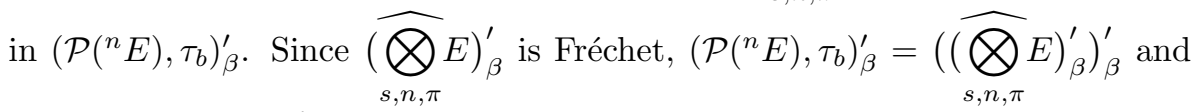
$\left(\mathcal{P}\left({ }^{n} E\right), \tau_{b}\right)_{i}^{\prime}=\left(\left(\widehat{\bigotimes}_{s, n, \pi} E\right)_{\beta}^{\prime}\right)_{i}^{\prime}$ have the same bounded sets ([11], Example 1.24). Hence $J_{n}^{b w}$ maps bounded sets onto bounded sets and by ([14], Proposition 3.7.1) is continuous.

Conversely, let $J_{n}^{b w}$ be continuous. Then $J_{n}^{b b}$ is continuous and $E$ is infrabarrelled by Remark 2 . When $n=1$ we obtain that $E_{\beta \beta}^{\prime \prime}=E_{\beta i}^{\prime \prime}$, and since inductive duals are barrelled, $E_{\beta \beta}^{\prime \prime}$ is barrelled and hence infrabarrelled. Moreover, since $\left(\mathcal{P}\left({ }^{n} E\right), \tau_{b}\right)=\left(\widehat{\bigotimes}_{s, n, \pi} E\right)_{\beta}^{\prime}$ is Fréchet, its inductive dual is complete ([15], Corollary 13.4.3). Hence we can extend $J_{n}^{b w}$ from $\bigotimes_{s, n, \pi} E_{\beta \beta}^{\prime \prime}$ to $\widehat{\bigotimes_{s, n, \pi}} E_{\beta \beta}^{\prime \prime}$ by continuity.

Now we consider polynomials on reflexive A-nuclear spaces (a number of these results also hold for fully nuclear spaces and for fully nuclear spaces with a basis). A locally convex space $E$ is $A$-nuclear if it has an absolute basis $\left(e_{n}\right)_{n}$ and there exists a sequence of positive real numbers $\left(\delta_{n}\right)_{n}, \sum_{i=1}^{\infty} \frac{1}{\delta_{i}}<\infty$, such that for each $p \in c s(E)$ the semi-norm

$$
q\left(\sum_{i=1}^{\infty} x_{i} e_{i}\right)=\sum_{i=1}^{\infty} \delta_{i} p\left(x_{i} e_{i}\right)
$$

is continuous. By the Grothendieck-Pietsch criterion every A-nuclear space is nuclear. Since the closed bounded subsets of a complete A-nuclear space $E$ are compact, $\tau_{0}=\tau_{b}$ on $\mathcal{P}\left({ }^{n} E\right)$ for every $n$. 
A polynomial $P \in \mathcal{P}\left({ }^{n} E\right)$ has finite rank if there exists a finite subset $\left\{\varphi_{i}\right\}_{i=1}^{l}$ in $E^{\prime}$ such that

$$
P(x)=\sum_{i=1}^{l} \varphi_{i}^{n}(x)
$$

for all $x \in E$. We let $\mathcal{P}_{f}\left({ }^{n} E\right)$ denote the space of all $n$-homogeneous polynomials of finite rank on E. By ([7], p. 186),

$$
\mathcal{P}_{f}\left({ }^{n} E\right)=\bigotimes_{s, n} E_{\beta}^{\prime}
$$

Polynomials in $\mathcal{P}_{A}\left({ }^{n} E\right)$, the closure of $\mathcal{P}_{f}\left({ }^{n} E\right)$ in $\left(\mathcal{P}\left({ }^{n} E\right), \tau_{b}\right)$, are called continuous approximable polynomials.

An element in $\mathcal{P}_{a}\left({ }^{n} E, F\right)$ is hypocontinuous if its restriction to each compact set is continuous. We let $\mathcal{P}_{H Y}\left({ }^{n} E, F\right)$ denote the vector space of all hypocontinuous $n$-homogeneous polynomials from $E$ into $F$.

Proposition 3.4. If $E$ is a reflexive A-nuclear space then Diagram (3.2) takes the following form:

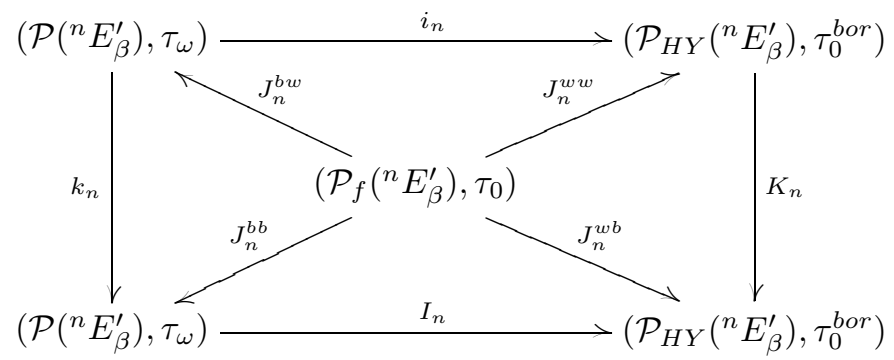

where $k_{n}$ and $K_{n}$ are isomorphisms.

Proof. If $E$ is a reflexive A-nuclear space then in a way similar to ([11], Proposition 3.46) it can be shown that $\left(\mathcal{P}\left({ }^{n} E\right), \tau_{0}\right)$ and $\left(\mathcal{P}\left({ }^{n} E\right), \tau_{\omega}\right)$ are Anuclear. Thus $\left(\overline{\mathcal{P}\left({ }^{n} E\right), \tau_{0}}\right)$ and $\left(\overline{\mathcal{P}\left({ }^{n} E\right), \tau_{\omega}}\right)$ are complete A-nuclear and, by [4], are reinforced regular, i.e. $\left(\overline{\mathcal{P}\left({ }^{n} E\right), \tau_{0}}\right)_{\beta}^{\prime}=\left(\overline{\mathcal{P}\left({ }^{n} E\right), \tau_{0}}\right)_{i}^{\prime}$ and $\left(\overline{\mathcal{P}\left({ }^{n} E\right), \tau_{\omega}}\right)_{\beta}^{\prime}=$ $\left(\overline{\mathcal{P}\left({ }^{n} E\right), \tau_{\omega}}\right)_{i}^{\prime}$. By $\quad\left([10]\right.$, Corollary 5.7) $\left(\overline{\mathcal{P}\left({ }^{n} E\right), \tau_{0}}\right)_{\beta}^{\prime}=\left(\mathcal{P}\left({ }^{n} E\right), \tau_{0}\right)_{\beta}^{\prime}$ and $\left(\overline{\mathcal{P}\left({ }^{n} E\right), \tau_{\omega}}\right)_{\beta}^{\prime}=\left(\mathcal{P}\left({ }^{n} E\right), \tau_{\omega}\right)_{\beta}^{\prime}$. Since similar equalities hold for the inductive duals ([15], p. 200), $k_{n}$ and $K_{n}$ are isomorphisms.

By ([10], Proposition 1.56) $\left(\mathcal{P}\left({ }^{n} E\right), \tau_{0}\right)_{\beta}^{\prime}=\left(\mathcal{P}\left({ }^{n} E_{\beta}^{\prime}\right), \tau_{\omega}\right)$ and by $([10]$, Proposition 1.48) the Borel transform is an algebraic isomorphism from 
$\left(\mathcal{P}\left({ }^{n} E\right), \tau_{\omega}\right)^{\prime}$ onto $\mathcal{P}_{H Y}\left({ }^{n} E_{\beta}^{\prime}\right)$ under which the equicontinuous subsets of $\left(\mathcal{P}\left({ }^{n} E\right), \tau_{\omega}\right)^{\prime}$ can be identified with the $\tau_{0}$-bounded subsets of $\mathcal{P}_{H Y}\left({ }^{n} E_{\beta}^{\prime}\right)$. Let $\left(\mathcal{P}\left({ }^{n} E\right), \tau_{\omega}\right)_{\beta}^{\prime}=\left(\mathcal{P}_{H Y}\left({ }^{n} E_{\beta}^{\prime}\right), \tau\right)$ for some topology $\tau$. Since $\left(\mathcal{P}\left({ }^{n} E\right), \tau_{\omega}\right)$ is barrelled, the equicontinuous subsets of its dual coincide with the $\tau$-bounded subsets of $\mathcal{P}_{H Y}\left({ }^{n} E_{\beta}^{\prime}\right)$. Hence $\tau$ and $\tau_{0}$ define the same bounded sets on $\mathcal{P}_{H Y}\left({ }^{n} E_{\beta}^{\prime}\right)$. Since $K_{n}$ is an isomorphism $\left(\mathcal{P}\left({ }^{n} E\right), \tau_{\omega}\right)_{\beta}^{\prime}$ is bornological and hence $\tau$ is the bornological topology associated with $\tau_{0}, \tau_{0}^{\text {bor }}$.

Finally, since $E$ is infrabarrelled $E_{e}^{\prime \prime}=E_{\beta \beta}^{\prime \prime}$, and, by (3.3), $\bigotimes_{s, n} E_{\beta \beta}^{\prime \prime}=$ $\mathcal{P}_{f}\left({ }^{n} E_{\beta}^{\prime}\right)$. As $E$ is a reflexive nuclear space, $\bigotimes_{s, n, \pi} E_{\beta \beta}^{\prime \prime}=\left(\mathcal{P}_{f}\left({ }^{n} E_{\beta}^{\prime}\right), \tau_{0}\right)([11]$, Proposition 2.13).

Let $E$ be a reflexive A-nuclear space and $n$ a positive integer. By Diagram (3.4) and the proof of Proposition 3.4 we have established the following identifications:

$$
\begin{aligned}
\left(\mathcal{P}\left({ }^{n} E\right), \tau_{\omega}\right)_{\beta}^{\prime} & =\left(\mathcal{P}\left({ }^{n} E\right), \tau_{w}\right)_{i}^{\prime}=\left(\mathcal{P}_{H Y}\left({ }^{n} E_{\beta}^{\prime}\right), \tau_{0}^{b o r}\right), \\
\left(\mathcal{P}\left({ }^{n} E\right), \tau_{0}\right)_{\beta}^{\prime} & =\left(\mathcal{P}\left({ }^{n} E\right), \tau_{0}\right)_{i}^{\prime}=\left(\mathcal{P}\left({ }^{n} E_{\beta}^{\prime}\right), \tau_{\omega}\right) .
\end{aligned}
$$

By (3.5), $\left(\mathcal{P}_{H Y}\left({ }^{n} E_{\beta}^{\prime}\right), \tau_{0}^{\text {bor }}\right)$ is complete as a strong dual of bornological space.

Corollary 3.1. Let $E$ be a reflexive A-nuclear space and $n$ a positive integer. Then

(a) $J_{n}^{w b}$ is continuous if and only if $\tau_{0}=\tau_{0}^{\text {bor }}$ on $\mathcal{P}_{H Y}\left({ }^{n} E_{\beta}^{\prime}\right)$.

(b) $J_{n}^{b w}$ is continuous if and only if $\tau_{0}=\tau_{\omega}$ on $\mathcal{P}\left({ }^{n} E_{\beta}^{\prime}\right)$.

(c) $I_{n}$ is an isomorphism if and only if $\tau_{0}=\tau_{\omega}$ on $\mathcal{P}\left({ }^{n} E\right)$.

Proof. (a) If $\tau_{0}=\tau_{0}^{\text {bor }}$ on $\mathcal{P}_{H Y}\left({ }^{n} E_{\beta}^{\prime}\right)$ then $J_{n}^{w b}$ is continuous by Diagram (3.4). Conversely, if $J_{n}^{w b}$ is continuous then it extends to a continuous mapping $\tilde{J}_{n}^{w b}$ from $\left(\mathcal{P}_{H Y}\left({ }^{n} E_{\beta}^{\prime}\right), \tau_{0}\right)$ into $\left(\mathcal{P}_{H Y}\left({ }^{n} E_{\beta}^{\prime}\right), \tau_{0}^{\text {bor }}\right)$. Since $J_{n}^{w b}(P)=P$ for all $P$ on a dense subspace of $\mathcal{P}_{H Y}\left({ }^{n} E_{\beta}^{\prime}\right)$, we have $\tilde{J}_{n}^{w b}(P)=P$ for all $P \in \mathcal{P}_{H Y}\left({ }^{n} E_{\beta}^{\prime}\right)$. Hence $\tau_{0}=\tau_{0}^{\text {bor }}$ on $\mathcal{P}_{H Y}\left({ }^{n} E_{\beta}^{\prime}\right)$.

(b) The method used for (a) can be adapted to prove (b). We give, however, an alternative proof. Clearly, by Diagram (3.4), if $\tau_{0}=\tau_{\omega}$ on $\mathcal{P}\left({ }^{n} E_{\beta}^{\prime}\right)$ then $J_{n}^{b w}$ is continuous. Conversely, let $J_{n}^{b w}$ be continuous. If $p$ is a $\tau_{\omega}$-continuous semi-norm on $\mathcal{P}\left({ }^{n} E_{\beta}^{\prime}\right)$ then there exist a compact polydisc $K \subset E_{\beta}^{\prime}$ such that $p(P) \leq\|P\|_{K}$ for all $P \in \mathcal{P}_{f}\left({ }^{n} E_{\beta}^{\prime}\right)$. If $\delta=\left(\delta_{n}\right)_{n}$ is the sequence defining 
A-nuclearity, as in $([11]$, p. 205) it can be shown that there exists $C(\delta)>0$ such that

$$
\sum_{m \in \mathbb{N}^{(\mathbb{N})},|m|=n}\left|a_{m}\right|\left\|z^{m}\right\|_{K} \leq C(\delta)\left\|\sum_{m \in \mathbb{N}^{(\mathbb{N})},|m|=n} a_{m} z^{m}\right\|_{\delta K}
$$

for all $\sum_{m \in \mathbb{N}^{(\mathbb{N})},|m|=n} a_{m} z^{m} \in \mathcal{P}\left({ }^{n} E_{\beta}^{\prime}\right)$. The set $\delta K$ is a compact polydisc in $E_{\beta}^{\prime}$. By the proof of ([11], Proposition 3.45) the semi-norm

$$
\tilde{p}\left(\sum_{m \in \mathbb{N}^{(\mathbb{N})},|m|=n} a_{m} z^{m}\right):=\sum_{m \in \mathbb{N}^{(\mathbb{N})},|m|=n}\left|a_{m}\right| p\left(z^{m}\right)
$$

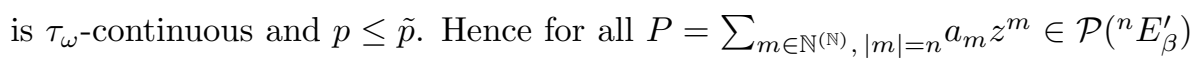
we have

$$
\begin{aligned}
p(P) \leq \tilde{p}(P) & =\sum_{m \in \mathbb{N}^{(\mathbb{N})},|m|=n}\left|a_{m}\right| p\left(z^{m}\right) \\
& \leq \sum_{m \in \mathbb{N}^{(\mathbb{N})},|m|=n}\left|a_{m}\right|\left\|z^{m}\right\|_{K} \leq C(\delta)\|P\|_{\delta K} .
\end{aligned}
$$

Hence $\tau_{\omega}=\tau_{0}$ on $\mathcal{P}\left({ }^{n} E_{\beta}^{\prime}\right)$.

(c) If $I_{n}$ is an isomorphism then, by Diagram (3.4), $\mathcal{P}\left({ }^{n} E_{\beta}^{\prime}\right)=\mathcal{P}_{H Y}\left({ }^{n} E_{\beta}^{\prime}\right)$. By ([10], Propositions 1.47 and 1.48) this implies $\left(\mathcal{P}\left({ }^{n} E\right), \tau_{0}\right)^{\prime}=\left(\mathcal{P}\left({ }^{n} E\right), \tau_{\omega}\right)^{\prime}$. Since the monomials form an absolute basis for both $\left(\mathcal{P}\left({ }^{n} E\right), \tau_{0}\right)$ and $\left(\mathcal{P}\left({ }^{n} E\right)\right.$, $\left.\tau_{\omega}\right)$, by $\left([11]\right.$, Lemma 4.41) $\tau_{0}=\tau_{\omega}$ on $\mathcal{P}\left({ }^{n} E\right)$.

Conversely, if $\tau_{0}=\tau_{\omega}$ on $\mathcal{P}\left({ }^{n} E\right)$ then, by Diagram (3.2), $I_{n}$ is an isomorphism.

Proposition 3.5. If $E$ is a reflexive A-nuclear space then the following are equivalent:

(a) $J_{n}^{w b}$ is continuous.

(b) The $\tau_{\omega}$-bounded sets of $\mathcal{P}\left({ }^{n} E\right)$ are locally bounded.

(c) $\left(\mathcal{P}\left({ }^{n} E\right), \tau_{\omega}\right)_{\beta}^{\prime}=\left(\mathcal{P}_{H Y}\left({ }^{n} E_{\beta}^{\prime}\right), \tau_{0}\right)$.

(d) $\left(\mathcal{P}\left({ }^{n} E\right), \tau_{\omega}\right)$ is quasi-complete.

(e) $\left(\mathcal{P}\left({ }^{n} E\right), \tau_{\omega}\right)$ is semi-reflexive.

Proof. If the $\tau_{\omega}$-bounded sets of $\mathcal{P}\left({ }^{n} E\right)$ are locally bounded then $J_{n}^{w b}$ is continuous by Proposition 3.1, hence (b) $\Rightarrow$ (a). 
Conversely, suppose $J_{n}^{w b}$ is continuous. By Corollary 3.1, $\tau_{0}=\tau_{0}^{\text {bor }}$ on $\mathcal{P}_{H Y}\left({ }^{n} E_{\beta}^{\prime}\right)$ and $(\mathrm{a}) \Rightarrow(\mathrm{c})$ by $(3.5)$.

(b) $\Leftrightarrow$ (c) follows from ([10], Proposition 1.57). By the proof of ([10], Proposition 5.37) conditions (b), (d) and (e) are equivalent.

Further equivalent conditions can be found in [12]. By Corollary 3.1(b) and the proof of ([11], Corollary 4.46) we obtain the following result.

Proposition 3.6. If $E$ is a reflexive A-nuclear space then the following are equivalent:

(a) $J_{n}^{b w}$ is continuous.

(b) The $\tau_{0}$-bounded sets of $\mathcal{P}\left({ }^{n} E\right)$ are locally bounded.

(c) $\mathcal{P}\left({ }^{n} E\right)=\mathcal{P}_{H Y}\left({ }^{n} E\right)$.

(d) $\left(\mathcal{P}\left({ }^{n} E\right), \tau_{0}\right)$ is complete.

Remark 3.

(a) Proposition 3.5 shows that the hypothesis in Proposition 3.1 is both necessary and sufficient when $E$ is a reflexive A-nuclear space.

(b) If $E$ is a reflexive A-nuclear space and $J_{n}^{w b}$ (respectively $J_{n}^{b w}$ ) is continuous, then it extends to define an isomorphism onto $\mathcal{P}_{H Y}\left({ }^{n} E_{\beta}^{\prime}\right)$ (respectively $\left.\mathcal{P}\left({ }^{n} E_{\beta}^{\prime}\right)\right)$.

(c) If $E$ is Fréchet nuclear (or DFN) with basis, then $E$ is a reflexive A-nuclear space and $\tau_{0}=\tau_{\omega}$ on $\mathcal{P}\left({ }^{n} E_{\beta}^{\prime}\right)$ for every $n$ ([11], Example 2.18). Hence both $J_{n}^{w b}$ and $J_{n}^{b w}$ extend to isomorphisms from the respective completions of their domains.

(d) Countable direct sums and products of reflexive A-nuclear spaces are again reflexive A-nuclear spaces.

\section{Example 1.}

1. Let $E=\prod_{k=1}^{\infty} E_{k}$ where each $E_{k}$ is a DFN space. Then $J_{n}^{w b}$ is always continuous and $J_{n}^{b w}$ is continuous if and only if each $\left(E_{k}\right)_{\beta}^{\prime}$ admits a continuous norm.

2. Let $E=\bigoplus_{k=1}^{\infty} E_{j}$ where each $E_{j}$ is Fréchet nuclear space with a basis. Then 
(a) $J_{n}^{w b}$ is continuous if and only if $E$ is isomorphic to one of the spaces $\mathbb{C}^{(\mathbb{N})}, \mathbb{C}^{(\mathbb{N})} \times \mathbb{C}^{\mathbb{N}}$ or $\left(\mathbb{C}^{\mathbb{N}}\right)^{(\mathbb{N})}$

(b) $J_{n}^{b w}$ is continuous if and only if $E$ is isomorphic to $\mathbb{C}^{(\mathbb{N})}$.

Proof. (1) The $\tau_{\omega}$-bounded subsets of $\mathcal{P}\left({ }^{n} E\right)$ are locally bounded ([11], Example 3.24(c)), hence by Proposition 3.5 $J_{n}^{w b}$ is continuous. By ([18], Proposition 2) $\mathcal{P}\left({ }^{n} E\right)=\mathcal{P}_{H Y}\left({ }^{n} E\right)$ if and only if there exists a continuous norm on $\left(E_{k}\right)_{\beta}^{\prime}$ for every $k$. It suffices to apply Proposition 3.6 to obtain the required result for $J_{n}^{b w}$.

(2) Part (a) follows from Proposition 3.5 and ([8], Theorem 1); part (b) follows from Proposition 3.6, ([8], Theorem 1) and ([11], Example 3.24(b)).

Example 2. Let $\mathcal{D}=\bigoplus_{k=1}^{\infty} s_{j}$ where each $s_{j}$ is the Fréchet nuclear space of rapidly decreasing sequences. By Example 1 neither of $J_{n}^{b w}$ or $J_{n}^{w b}$ are continuous. By ([6], Proposition 9$) \tau_{0}=\tau_{\omega}$ on $\mathcal{P}\left({ }^{n} \mathcal{D}\right)$, hence, by Corollary, 3.1(c) $I_{n}$ is an isomorphism.

\section{$\S 4$. Continuity of $\boldsymbol{J}_{n}$}

In Section 3 we concentrated on continuity of the mappings $J_{n}^{*}$. In this section we discuss injectivity. Let $x^{\prime \prime} \in E_{e}^{\prime \prime}$ and $\varphi \in E^{\prime}$. Then $\otimes_{n} x^{\prime \prime} \in \bigotimes_{s, n, \pi} E_{e}^{\prime \prime}$, $\varphi^{n} \in \mathcal{P}_{f}\left({ }^{n} E\right)$, and we have the duality

$$
\left\langle\otimes_{n} x^{\prime \prime}, \varphi^{n}\right\rangle=x^{\prime \prime}(\varphi)^{n} .
$$

Suppose

$$
\left[J_{n}\left(\otimes_{n} x^{\prime \prime}\right)\right]\left(\varphi^{n}\right)=0
$$

for every $\varphi \in E^{\prime}$. Then, by (4.1), $x^{\prime \prime}(\varphi)^{n}=0$ for all $x^{\prime \prime} \in E_{e}^{\prime \prime}$ and hence $\otimes_{n} x^{\prime \prime}=0$. This motivates us to restrict our attention to $\mathcal{P}_{f}\left({ }^{n} E\right)$, and our results in Section 5 show that this is indeed a good choice. Let $R(T):=\left.T\right|_{\mathcal{P}_{f}\left({ }^{n} E\right)}$ for $T \in\left(\mathcal{P}\left({ }^{n} E\right), \tau_{b}\right)^{\prime}$. We let $J_{n}^{f}:=R \circ J_{n}^{b w}$. By ([15], Proposition 10.3.4)

$$
R:\left(\mathcal{P}\left({ }^{n} E\right), \tau_{b}\right)_{i}^{\prime} \longrightarrow\left(\mathcal{P}_{f}\left({ }^{n} E\right), \tau_{b}\right)_{i}^{\prime}
$$

is continuous and open, hence if $J_{n}^{b w}$ is continuous then $J_{n}^{f}$ is continuous.

In order to investigate the continuity of $J_{n}^{f}$ we require some further definitions. An $n$-homogeneous polynomial $P$ on $E$ is called nuclear if there exist an equicontinuous sequence $\left(\psi_{i}\right)_{i}$ in $E^{\prime}$ and $\left(\lambda_{i}\right)_{i}$ in $l_{1}$ such that

$$
P(x)=\sum_{i=1}^{\infty} \lambda_{i} \psi_{i}{ }^{n}(x)
$$


for all $x$ in $E$. Let $\mathcal{P}_{N}\left({ }^{n} E\right)$ denote the space of all nuclear polynomials on $E$. If $A$ is a subset of $E$ let

$$
\pi_{N, A}(P)=\|P\|_{N, A}:=\inf \left[\sum_{i=1}^{\infty}\left|\lambda_{i}\right|\left\|\psi_{i}\right\|_{A}^{n}: P=\sum_{i=1}^{\infty} \lambda_{i} \psi_{i}^{n}\right]
$$

As $A$ ranges over the bounded sets of $E$ we obtain the $\pi_{b}$ topology. We also let

$$
\left(\mathcal{P}_{N}\left({ }^{n} E\right), \pi_{\omega}\right)=\underset{\alpha \in \overrightarrow{c s}(E)}{\lim }\left(\mathcal{P}_{N}\left({ }^{n} E_{\alpha}\right), \pi_{b}\right)
$$

The space of all $n$-homogeneous (algebraic) polynomials on $E^{\prime}$ which are bounded on the equicontinuous subsets of $E^{\prime}$ is denoted by $\mathcal{P}_{\xi}\left({ }^{n} E^{\prime}\right)$. An $n$ homogeneous polynomial $P$ on a locally convex space $E$ is integral if there is an absolutely convex closed neighbourhood of $0, U$, and a finite regular Borel measure $\mu$ on $U^{\circ}$ endowed with the $w^{*}$-topology, such that

$$
P(x)=\int_{U^{\circ}} \psi^{n}(x) d \mu(\psi)
$$

for all $x \in E$. The space of all $n$-homogeneous integral polynomials on $E$ is denoted by $\mathcal{P}_{I}\left({ }^{n} E\right)$, and the topology $\tau_{I}$ is defined as the locally convex inductive limit

$$
\left(\mathcal{P}_{I}\left({ }^{n} E\right), \tau_{I}\right)=\underset{U \in \mathcal{U}}{\lim }\left(\mathcal{P}\left({ }^{n} E_{U}\right),\|\cdot\|_{U, I}\right)
$$

where

$$
\|P\|_{U, I}=\inf \left\{\|\mu\|_{U^{\circ}}: P(x)=\int_{U^{\circ}} \psi^{n}(x) d \mu(\psi)\right\} .
$$

Clearly every polynomial of finite rank is nuclear, hence $\mathcal{P}_{f}\left({ }^{n} E\right)$ is a subset of both $\mathcal{P}_{N}\left({ }^{n} E\right)$ and $\mathcal{P}_{I}\left({ }^{n} E\right)$. Moreover, by ([7], p. 186) the algebraic representation (3.3) can be extended to give

$$
\left(\mathcal{P}_{f}\left({ }^{n} E\right), \pi_{b}\right)=\bigotimes_{s, n, \pi} E_{\beta}^{\prime} .
$$

The space $\mathcal{P}_{f}\left({ }^{n} E\right)$ is dense both in $\left(\mathcal{P}_{N}\left({ }^{n} E\right), \pi_{b}\right)$ and $\left(\mathcal{P}_{N}\left({ }^{n} E\right), \pi_{w}\right)$. This often allows us to use finite polynomials in place of nuclear polynomials and to avoid the approximation property. Clearly $\pi_{\omega} \geq \pi_{b}$ and, since in the Banach space case $\|\cdot\|_{I} \leq\|\cdot\|_{N}$, the topology $\pi_{\omega}$ is finer than $\tau_{I}$.

Lemma 4.1. Let $E$ be an infrabarrelled locally convex space and $n$ be a positive integer. The mapping $J_{n}^{f}$ is continuous if and only if $\pi_{b}$ is finer then $\tau_{I}$ on $\mathcal{P}_{f}\left({ }^{n} E_{\beta}^{\prime}\right)$. 
Proof. By ([7], Proposition 2)

$$
\left(\mathcal{P}_{f}\left({ }^{n} E\right), \tau_{b}\right)_{i}^{\prime}=\left(\mathcal{P}_{A}\left({ }^{n} E\right), \tau_{b}\right)_{i}^{\prime}=\left(\mathcal{P}_{I}\left({ }^{n} E_{\beta}^{\prime}\right), \tau_{I}\right) .
$$

By (4.2), $\bigotimes_{s, n, \pi} E_{\beta \beta}^{\prime \prime}=\left(\mathcal{P}_{f}\left({ }^{n} E_{\beta}^{\prime}\right), \pi_{b}\right)$, and hence $J_{n}^{f}$ is the identity mapping

$$
\left(\mathcal{P}_{f}\left({ }^{n} E_{\beta}^{\prime}\right), \pi_{b}\right) \longrightarrow\left(\mathcal{P}_{f}\left({ }^{n} E_{\beta}^{\prime}\right), \tau_{I}\right)
$$

This completes the proof.

A locally convex space $E$ is locally Asplund if for every probability space $(\Omega, \Sigma, \mu)$ all operators $T: L^{1}(\mu) \rightarrow E^{\prime}$ which map some neighbourhood of 0 into an equicontinuous set are locally representable. By [9] locally Asplund spaces include Schwartz spaces, reflexive quasinormable spaces and DF spaces with separable duals. By [7] if $E$ is locally Asplund then $\left(\mathcal{P}_{I}\left({ }^{n} E\right), \tau_{I}\right)=$ $\left(\mathcal{P}_{N}\left({ }^{n} E\right), \pi_{w}\right)$. By Lemma 4.1 this implies the following result.

Corollary 4.1. If $E$ is an infrabarrelled locally convex space and $E_{\beta}^{\prime}$ is locally Asplund, then $J_{n}^{f}$ is continuous if and only if $\pi_{w}=\pi_{b}$ on $\mathcal{P}_{f}\left({ }^{n} E_{\beta}^{\prime}\right)$.

Proposition 4.1. If $E$ is an infrabarrelled locally convex space. Then $\pi_{w}=\pi_{b}$ on $\mathcal{P}_{f}\left({ }^{n} E_{\beta}^{\prime}\right)$ if and only if $\mathcal{P}_{\xi}\left({ }^{n} E_{\beta \beta}^{\prime \prime}\right)=\mathcal{P}\left({ }^{n} E_{\beta \beta}^{\prime \prime}\right)$ and the subsets of $\mathcal{P}\left({ }^{n} E_{\beta \beta}^{\prime \prime}\right)$ which are bounded on the equicontinuous subsets of $E_{\beta \beta}^{\prime \prime}$ are locally bounded.

If these conditions are satisfied, $J_{n}^{f}$ is continuous for every $n$.

Proof. See [10], Propositions 1.47 and 1.48. The final remark follows from Lemma 4.1.

Proposition 4.2. Let $E$ be a Fréchet space, then $J_{n}^{f}$ is continuous for every positive integer $n$ and extends to $\widehat{\bigotimes_{s, n, \pi}} E_{\beta \beta}^{\prime \prime}$.

Proof. If $E$ is Fréchet then $E_{\beta \beta}^{\prime \prime}$ is Fréchet. Thus every convergent sequence in $E_{\beta \beta}^{\prime \prime}$ is equicontinuous (see [14], p. 293), hence $\mathcal{P}_{\xi}\left({ }^{n} E_{\beta \beta}^{\prime \prime}\right)=\mathcal{P}\left({ }^{n} E_{\beta \beta}^{\prime \prime}\right)$. Moreover, subsets of $\mathcal{P}\left({ }^{n} E_{\beta \beta}^{\prime \prime}\right)$ which are bounded on convergent sequences in $E_{\beta \beta}^{\prime \prime}$ are locally bounded by ([11], Example 1.24). By Proposition 4.1 this implies the continuity of $J_{n}^{f}$.

Let $\theta \in \widehat{\bigotimes}_{s, n, \pi} E_{\beta \beta}^{\prime \prime}$, the completion of $\bigotimes_{s, n, \pi} E_{\beta \beta}^{\prime \prime}$. Then $\theta$ has a representation $\sum_{i=1}^{\infty} \lambda_{i} \otimes_{n} x_{i}$, where $\left(x_{i}\right)_{i}$ is a null sequence in $E_{\beta \beta}^{\prime \prime}$ and $\left(\lambda_{i}\right)_{i} \in l_{1}$ 
([15], Corollary 15.6.4). Since $\left(x_{i}\right)_{i}$ is a countable bounded subset of $E_{\beta \beta}^{\prime \prime}$, it is equicontinuous and hence there exists a bounded subset $B$ in $E$ such that $\left(x_{i}\right)_{i} \subset B^{\circ \circ}$. By ([13], Theorem 1.5)

$$
\left|\left(J_{n}^{f}\left(\otimes_{n} x_{i}\right)\right)(P)\right| \leq\left\|A B_{n}(P)\right\|_{B^{\circ \circ} \leq\|P\|_{B}}
$$

and consequently for each $i \in \mathbb{N}, J_{n}^{f}\left(\otimes_{n} x_{i}\right)$ lies in $\left(P \in \mathcal{P}_{f}\left({ }^{n} E\right):\|P\|_{B} \leq 1\right)^{\circ}$. Therefore $J_{n}^{f}\left(\sum_{i=1}^{\infty} \lambda_{i} \otimes_{n} x_{i}\right)$ belongs to $\left(\mathcal{P}_{f}\left({ }^{n} E\right), \tau_{b}\right)_{i}^{\prime}$. This completes the proof.

\section{§5. Definition and Basic Properties of Q-reflexive Locally Convex Spaces}

In this section we define Q-reflexive locally convex spaces and discuss their basic properties.

Definition 5.1. The locally convex space $E$ is $Q$-reflexive if for every positive integer $n$ :

1. The mapping $J_{n}^{b w}$ is continuous.

2. The extension $J_{n}$ of $J_{n}^{b w}$ to the completion is an isomorphism between $\bigotimes_{s, n, \pi} E_{e}^{\prime \prime}$ and $\overline{\left(\mathcal{P}\left({ }^{n} E\right), \tau_{b}\right)_{i}^{\prime}}$.

By Remark 2 every locally convex Q-reflexive space $E$ is infrabarrelled.

A locally convex space $E$ has the strict approximation property if it admits a fundamental system $\mathcal{A}$ of semi-norms such that $E_{\alpha}=(E, \alpha) / \alpha^{-1}(0)$ has the approximation property for each $\alpha \in \mathcal{A}$.

Proposition 5.1. If $E$ is an infrabarrelled locally convex space whose strong bidual has the strict approximation property, then the following conditions are equivalent:

1. E is Q-reflexive.

2. $\overline{\left(\mathcal{P}_{N}\left({ }^{n} E_{\beta}^{\prime}\right), \pi_{b}\right)}=\left(\mathcal{P}_{I}\left({ }^{n} E_{\beta}^{\prime}\right), \tau_{I}\right)$ and $\mathcal{P}\left({ }^{n} E\right)=\mathcal{P}_{A}\left({ }^{n} E\right)$ for every positive integer $n$.

Proof. $\quad(\mathbf{1}) \Rightarrow(\mathbf{2})$ Since $J_{n}^{b w}$ is continuous, $J_{n}^{f}$ is continuous and can be extended to a mapping

$$
\bar{J}_{n}^{f}: \widehat{\bigotimes}_{s, n, \pi} E_{e}^{\prime \prime} \longrightarrow \overline{\left(\mathcal{P}_{f}\left({ }^{n} E\right), \tau_{b}\right)_{i}^{\prime}} .
$$


Suppose $\mathcal{P}_{A}\left({ }^{n} E\right) \neq \mathcal{P}\left({ }^{n} E\right)$. By the Hahn-Banach Theorem there exists a nonzero functional $\varphi \in\left(\mathcal{P}\left({ }^{n} E\right), \tau_{b}\right)^{\prime}$ such that $\left.\varphi\right|_{\mathcal{P}_{f}\left({ }^{n} E\right)}=0$. Since $E$ is Q-reflexive there exists $z \in \widehat{\bigotimes_{s, n, \pi}} E_{e}^{\prime \prime}$ such that $\bar{J}_{n}^{f}(z)=\left.\varphi\right|_{\mathcal{P}_{f}\left({ }^{n} E\right)}=0$. Since $E_{\beta \beta}^{\prime \prime}=E_{e}^{\prime \prime}$ has the strict approximation property, $E$ has a neighbourhood basis at the origin, $\mathcal{U}$, consisting of convex open balanced sets such that $E_{U^{\circ \circ}}^{\prime \prime}$ has the approximation property for all $U \in \mathcal{U}$. The space $E$ can be written as $\underset{U \in \mathcal{U}}{\lim } E_{U}$. Then $E_{\beta \beta}^{\prime \prime}=\lim _{U \in \mathcal{U}} E_{U^{\circ \circ}}^{\prime \prime}$, so for every $U \in \mathcal{U}$ there exists a sequence $\left(x_{i}\right)_{i}$ in $E_{U^{\circ \circ}}^{\prime \prime}$ such that $z=\sum_{i=1}^{\infty} \otimes_{n} x_{i}$ and $\sum_{i=1}^{\infty}\left(\left\|x_{i}\right\|_{U^{\circ \circ}}\right)^{n}<\infty$. For all $\xi \in\left(E_{U}\right)^{\prime}$ we have

$$
\left[J_{n}^{f}(z)\right]\left(\xi^{n}\right)=\sum_{i=1}^{\infty} x_{i}^{n}(\xi)=0 .
$$

By Goldstine's Theorem for all $\psi \in\left(E_{U^{\circ \circ}}^{\prime \prime}\right)^{\prime}$

$$
\sum_{i=1}^{\infty}\left(\psi\left(x_{i}\right)\right)^{n}=0
$$

Hence $\|z\|_{U^{\circ}}=0$ for every $U \in \mathcal{U}$. As each $E_{U^{\circ}}^{\prime \prime}$ has the approximation property, this implies $z=0$ in $\widehat{\bigotimes_{s, n, \pi}} E_{\beta \beta}^{\prime \prime}$, hence $J_{n}(z)=\varphi=0$. This contradicts our choice of $\varphi$ and implies $\mathcal{P}_{A}\left({ }^{n} E\right)=\mathcal{P}\left({ }^{n} E\right)$.

Using Q-reflexivity and ([7], Proposition 2),

$$
\left(\mathcal{P}_{I}\left({ }^{n} E_{\beta}^{\prime}\right), \tau_{I}\right)=\left(\overline{\mathcal{P}_{A}\left({ }^{n} E\right), \tau_{b}}\right)_{i}^{\prime}=\left(\overline{\mathcal{P}\left({ }^{n} E\right), \tau_{b}}\right)_{i}^{\prime}=\widehat{\bigotimes_{s, n, \pi} E_{\beta \beta}^{\prime \prime}}=\overline{\left(\mathcal{P}_{N}\left({ }^{n} E_{\beta}^{\prime}\right), \pi_{b}\right)}
$$

(2) $\Rightarrow(\mathbf{1})$ By hypothesis

$$
\left(\mathcal{P}\left({ }^{n} E\right), \tau_{b}\right)_{i}^{\prime}=\left(\overline{\mathcal{P}_{A}\left({ }^{n} E\right), \tau_{b}}\right)_{i}^{\prime}=\left(\mathcal{P}_{I}\left({ }^{n} E_{\beta}^{\prime}\right), \tau_{I}\right)=\overline{\left(\mathcal{P}_{N}\left({ }^{n} E_{\beta}^{\prime}\right), \pi_{b}\right)}=\widehat{\bigotimes_{s, n, \pi}} E_{\beta \beta}^{\prime \prime} .
$$

Corollary 5.1. If $E$ is a $Q$-reflexive locally convex space whose strong bidual has the strict approximation property, then $J_{n}^{f}=J_{n}^{b w}$ for every positive integer $n$.

Next we list some properties of Q-reflexive spaces. The proofs can be found in $[19]$. 
Proposition 5.2. Let $E$ be a Q-reflexive locally convex space whose strong bidual has the strict approximation property. Then

(a) $l_{1}$ is not a subspace of $E_{\beta}^{\prime}$ or $E$.

(b) If $E$ is complete then $E_{\beta}^{\prime}$ does not contain a copy of $c_{0}$.

(c) If $E_{\beta}^{\prime}$ is barrelled then $E$ does not contain a copy of $c_{0}$.

(d) If $E$ is a complete DF space or a Fréchet space with $(B B)_{n}$ for every $n$, then $l_{\infty}$ is not a subspace of $\left(\mathcal{P}\left({ }^{n} E\right), \tau_{b}\right)$ for any $n$.

\section{§6. Examples of Q-reflexive Spaces}

In this section we give some examples of Q-reflexive locally convex spaces. Further examples are given in [19].

Every Q-reflexive Banach space satisfies Definition 5.1. On the other end of the spectrum, Fréchet nuclear and DFN spaces with a basis are Q-reflexive by Remark 3(c). This also is a special case of the following proposition.

Proposition 6.1. $\quad$ Let $E$ be a Fréchet-Montel space with $(B B)_{n}$ for every $n$. Then $E$ is Q-reflexive.

Proof. Since $E$ is Fréchet-Montel it is reflexive, hence $\widehat{\bigotimes_{s, n, \pi}} E_{\beta \beta}^{\prime \prime}=\widehat{\bigotimes_{s, n, \pi} E}$ for every $n$. By $\left([11]\right.$, Proposition 1.35) $\left(\mathcal{P}\left({ }^{n} E\right), \tau_{b}\right)={\widehat{\left(\bigotimes_{s, n, \pi}^{s, n, \pi} E\right)_{\beta}^{\prime}}}^{{ }^{s, n, \pi}}$ space and in particular is reflexive and reinforced regular. Hence

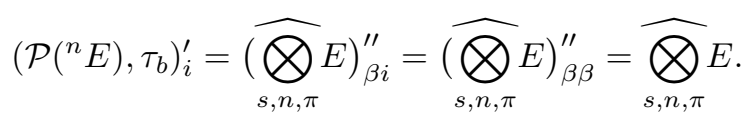

Proposition 6.2. The space $\mathbb{C}^{(I)}$ is Q-reflexive if and only if $I$ is countable.

Proof. Every bounded subset of $\mathbb{C}^{(I)}$ is finite dimensional and consequently every polynomial on $\mathbb{C}^{(I)}$ is continuous on bounded sets. The nuclear space $\mathbb{C}^{I}$ is locally Asplund and consequently, by ([7], Theorem 3$),\left(\mathcal{P}_{N}\left({ }^{n} \mathbb{C}^{I}\right)\right.$, $\left.\pi_{\omega}\right)=\left(\mathcal{P}_{I}\left({ }^{n} \mathbb{C}^{I}\right), \tau_{I}\right)$ for every $n$. Hence, by Proposition 5.1, $\mathbb{C}^{(I)}$ is Q-reflexive if and only if $\pi_{\omega}=\pi_{b}$ on $\mathcal{P}_{N}\left({ }^{n} \mathbb{C}^{I}\right)$. If $\left(\mathcal{P}_{N}\left({ }^{n} \mathbb{C}^{I}\right), \pi_{\omega}\right)=\left(\mathcal{P}_{N}\left({ }^{n} \mathbb{C}^{I}\right), \pi_{b}\right)$ then 
their duals will coincide, i.e. $\mathcal{P}_{\xi}\left({ }^{n} \mathbb{C}^{(I)}\right)=\mathcal{P}\left({ }^{n} \mathbb{C}^{(I)}\right)$. Since $\mathbb{C}^{I}$ is barrelled, the equicontinuous and the bounded sets of $\mathbb{C}^{(I)}$ coincide, i.e. all equicontinuous sets are finite dimensional. Thus $\mathcal{P}_{\xi}\left({ }^{n} \mathbb{C}^{(I)}\right)=\mathcal{P}_{a}\left({ }^{n} \mathbb{C}^{(I)}\right)$ and therefore $\mathcal{P}\left({ }^{n} \mathbb{C}^{(I)}\right)=\mathcal{P}_{a}\left({ }^{n} \mathbb{C}^{(I)}\right)$, the space of all $n$-homogeneous (algebraic) polynomials on $\mathbb{C}^{(I)}$. Since $\mathcal{P}\left({ }^{n} E\right)=\mathcal{P}_{a}\left({ }^{n} E\right)$ if and only if $E=\mathbb{C}^{(\mathbb{N})}, \mathbb{C}^{(I)}$ is Q-reflexive if and only if $\mathbb{C}^{(I)}=\mathbb{C}^{(\mathbb{N})}$.

The example $\mathbb{C}^{(\mathbb{N})} \times \mathbb{C}^{\mathbb{N}}$ shows that Q-reflexivity is not in general preserved by taking inductive or projective limits, direct sums or products. Indeed, $\mathbb{C}^{(\mathbb{N})} \times$ $\mathbb{C}^{\mathbb{N}}$ is both a countable direct sum of Q-reflexive Fréchet spaces and a countable product of Q-reflexive DF spaces, but is not Q-reflexive. The following example shows that Q-reflexivity is preserved in the case of the Tsirelson-James space $T_{J}{ }^{*}$.

Example 3. The direct sum $E:=\bigoplus_{k=1}^{\infty} T_{J}{ }^{*}$ and the product $F:=$ $\prod_{k=1}^{\infty} T_{J}^{*}$ are Q-reflexive spaces.

Proof. We note first that $E_{\beta \beta}^{\prime \prime}$ and $F_{\beta \beta}^{\prime \prime}$ have the strict approximation property (see [3]). Let $\left(T_{J}{ }^{*}\right)^{k}:=\underbrace{T_{J}{ }^{*} \times \cdots \times T_{J}^{*}}_{k}$. By ([20], Proposition 2.5.2), $\left(T_{J}^{*}\right)^{k}$ is a Q-reflexive Banach space. The space $\left(\left(T_{J}^{*}\right)^{k}\right)^{\prime}=$ $\underbrace{\left(T_{J}{ }^{*}\right)^{\prime} \times \cdots \times\left(T_{J}^{*}\right)^{\prime}}_{k}$ is Asplund and consequently locally Asplund. Since

$$
E_{\beta}^{\prime}=\prod_{k=1}^{\infty}\left(T_{J}^{*}\right)^{\prime}=\underset{\overleftarrow{k}}{\lim }\left(\left(T_{J}^{*}\right)^{k}\right)^{\prime},
$$

and projective limits of locally Asplund spaces are locally Asplund (see [9]), by ([7], Theorem 3) $\left(\mathcal{P}_{N}\left({ }^{n} E_{\beta}^{\prime}\right), \pi_{w}\right)=\left(\mathcal{P}_{I}\left({ }^{n} E_{\beta}^{\prime}\right), \tau_{I}\right)$ for every $n$. As a countable inductive limit of Banach spaces $E_{\beta \beta}^{\prime \prime}$ is a barrelled DF space, hence $E_{\beta}^{\prime}$ is a distinguished Fréchet space and by ([10], Corollary 1.53) $\pi_{w}=\pi_{b}$ on $\mathcal{P}_{N}\left({ }^{n} E_{\beta}^{\prime}\right)$. Hence $\left(\mathcal{P}_{N}\left({ }^{n} E_{\beta}^{\prime}\right), \pi_{b}\right)=\left(\mathcal{P}_{I}\left({ }^{n} E_{\beta}^{\prime}\right), \tau_{I}\right)$.

Let $P \in \mathcal{P}\left({ }^{n} E\right)$ and $B$ be a bounded subset of $E$. The countable strict inductive limit $\left.\underset{k}{\lim } \widehat{\left(\bigotimes_{n, \pi}\right.}\left(T_{J}{ }^{*}\right)^{k}\right)$ is regular, hence there exists positive integer $k$ such that $B \subset\left(T_{J}{ }^{*}\right)^{k}$. Since $\left(T_{J}{ }^{*}\right)^{k}$ is Q-reflexive $\mathcal{P}\left({ }^{n}\left(T_{J}{ }^{*}\right)^{k}\right)=\mathcal{P}_{A}\left({ }^{n}\left(T_{J}{ }^{*}\right)^{k}\right)$, hence for every $\epsilon>0$ we can find $R \in \mathcal{P}_{f}\left({ }^{n}\left(T_{J}{ }^{*}\right)^{k}\right)$ such that

$$
\left\|R-\left.P\right|_{\left(T_{J}^{*}\right)^{k}}\right\|_{B}<\epsilon
$$


Let $\tilde{R}(x+y):=R(x)$, where $x \in\left(T_{J}^{*}\right)^{k}$ and $y$ belongs to the complement of $\left(T_{J}{ }^{*}\right)^{k}$ in $E$. Then $\tilde{R} \in \mathcal{P}_{f}\left({ }^{n} E\right)$ and

$$
\|\tilde{R}-P\|_{B}=\left\|R-\left.P\right|_{\left(T_{J}^{*}\right)^{k}}\right\|_{B}<\epsilon .
$$

Hence $\mathcal{P}\left({ }^{n} E\right)=\mathcal{P}_{A}\left({ }^{n} E\right)$ and, by Proposition 5.1, $E$ is Q-reflexive.

Since the countable inductive limit of locally Asplund spaces is locally Asplund ([9]), $F_{\beta}^{\prime}$ is locally Asplund, therefore by ([7], Theorem 3) $\left(\mathcal{P}_{N}\left({ }^{n} F_{\beta}^{\prime}\right)\right.$, $\left.\pi_{\omega}\right)=\left(\mathcal{P}_{I}\left({ }^{n} F_{\beta}^{\prime}\right), \tau_{I}\right)$. As a countable inductive limit of Banach spaces $F_{\beta}^{\prime}$ is a barrelled DF space, hence by ([10], Corollary 1.53) $\pi_{\omega}=\pi_{b}$ on $\mathcal{P}_{N}\left({ }^{n} F_{\beta}^{\prime}\right)$. Thus $\left(\mathcal{P}_{N}\left({ }^{n} F_{\beta}^{\prime}\right), \pi_{b}\right)=\left(\mathcal{P}_{I}\left({ }^{n} F_{\beta}^{\prime}\right), \tau_{I}\right)$.

The implication $\mathcal{P}\left({ }^{n} F\right)=P_{A}\left({ }^{n} F\right)$ can be proved in a way similar to that used for $E$, where in place of the fact that the bounded subsets of $E$ are contained in a finite product we can use the fact that every continuous polynomial factors through $\left(T_{J}^{*}\right)^{k}$ for some integer $k$. Here we give an alternative proof.

By $([17], 44.5 .6) E \widehat{\bigotimes}_{\epsilon}\left(\bigoplus_{j=1}^{\infty} E_{j}\right)$ and $\bigoplus_{j=1}^{\infty}\left(E \widehat{\bigotimes}_{\epsilon} E_{j}\right)$ are isomorphic. Using this result $n$ times and applying ([15], Theorem 8.8.5) we obtain

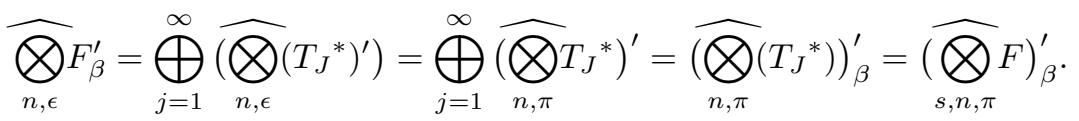

Applying the symmetrization operator we obtain $\mathcal{P}\left({ }^{n} F\right)=\widehat{\bigotimes} F_{\beta, n, \epsilon}^{\prime}=P_{A}\left({ }^{n} F\right)$. By Proposition 5.1 the proof is complete.

\section{References}

[1] Ansemil, J. M. and Ponte, S., The compact open topology and the Nachbin ported topology on spaces of holomorphic functions, Arch. Math. (Basel), 51 (1988), 65-70.

[2] Aron, R. M. and Berner, P., A Hahn-Banach extension theorem for analytic mappings, Bull. Soc. Math. France, 106 (2) (1978), 3-24.

[3] Aron, R. M. and Dineen, S., Q-reflexive Banach spaces, Rocky Mountain J. Math., 27 (4) (1997), 1009-1025.

[4] Berezanskii, I. A., Inductively reflexive locally convex spaces, Dokl. Akad. Nauk SSSR, 182 (1) (1968), 1080-1082.

[5] Bierstedt, K. D., An introduction to locally convex inductive limits, Funct. Anal. Appl., World Scientific Publishing, Singapore (1988), 35-133.

[6] Boland, P. and Dineen, S., Holomorphy on spaces of distributions, Pacific J. Math., 92 (1) (1981), 27-34.

[7] Boyd, C., Duality and reflexivity of spaces of approximable polynomials on locally convex spaces, Monatsh. Math., 130 (2000), 177-188.

[8] Boyd, C. and Dineen, S., Locally bounded subsets of holomorphic functions, Comp. Appl. Math., 13 (3) (1994), 189-194. 
[9] Defant, A., The local Radon Nikodým property for duals of locally convex spaces, Bulletin de la Société Royale des Sciencies de Liège, 53e année, 5 (1984), 233-246.

[10] Dineen, S., Complex analysis on locally convex spaces, North-Holland Math. Stud., 57 (1981).

[11] Complex analysis on infinite dimensional spaces, Monog. Math., SpringerVerlag, 1999.

[12] Dineen, S., Galindo, P., García, D. and Maestre, M., Linearization of holomorphic mappings on fully nuclear spaces with a basis, Glasgow Math. J., 36 (1994), 201-208

[13] Galindo, P., García, D. and Maestre, M., Entire functions of bounded type on Fréchet spaces, Math. Nachr., 161 (1993), 185-198.

[14] Horváth, J., Topological vector spaces and distributions, Vol. 1, Addison-Wesley, Massachusetts, 1966.

[15] Jarchow, H., Locally convex spaces, B.G. Teubner, 1981.

[16] Köthe, G., Topological vector spaces I, Springer, London-Berlin-Heidelberg, 1969.

[17] , Topological vector spaces II, Springer, London-Berlin-Heidelberg, 1979.

[18] Moraes, L., Holomorphic functions on strict inductive limits, Results Math., 4 (1981), 201-212.

[19] Venkova, M., Properties of Q-reflexive locally convex spaces, J. Korean Math. Soc., to appear.

[20] _ Q-reflexive locally convex spaces, Thesis, University College Dublin, 2002. 